\title{
Dynamic Minkowski Sum of Convex Shapes
}

Evan Behar

\begin{abstract}
Computing the Minkowski sums of rotating objects has always been done naïvely by re-computing every Minkowski sum from scratch. The correspondences between the Minkowski sums are typically completely ignored. We propose a method, called DYMSUM, that can efficiently update the Minkowski sums of rotating convex polyhedra. We show that DYMSUM is significantly more efficient than the traditional approach, in particular when the size of the input polyhedra are large and when the rotation is small between frames. From our experimental results, we show that the computation time of the proposed method grows slowly with respect to the size of the input comparing to the naïve approach.
\end{abstract}

\section{INTRODUCTION}

The Minkowski sum is an important operation in robotics due to its fundamental role in providing the geometricreasoning ability to the robots, such as configuration space mapping, collision detection, and penetration depth estimation. The Minkowski sum of two shapes $P$ and $Q$ is:

$$
P \oplus Q=\{p+q \mid p \in P, q \in Q\} .
$$

Although the problem of computing Minkowski sums has been studied since the early 70's, researchers have been paying more attention to this problem recently; see the surveys in [1], [2], [3]. In particular, in 3-dimensions, methods [4], [3], [5], [6] are known to compute the Minkowski sum of convex polyhedra efficiently.

In this work, we are interested in a method that can efficiently compute the Minkowski sum of rotating convex polyhedra. Computing the Minkowski sum of polyhedra undergoing rotations can be found in many problems, such as general penetration depth estimation [7] for physicallybased simulation and configuration-space obstacle mapping [8] for robotic motion planning. Figure 1 shows an example of the Minkowski sums before and after rotating the ellipse.

The main challenge of computing the Minkowski sum of two rotating polyhedra comes from that fact that the Minkowski sum can be dramatically different after the input polyhedra rotate. Therefore, existing methods simply recompute a new Minkowski sum every time $P$ or $Q$ rotates. For example, this approach is traditionally used to slice the $\mathcal{C}$-space obstacles $(\mathcal{C}$-obst $)$ in motion planning. When the rotation of the robot is considered, $\mathcal{C}$-obst are approximated by repetitively computing the Minkowski sums of the robot with different orientations. These Minkowski sums are usually separated by a fixed rotational resolution. A main problem of re-computing the Minkowski sum from scratch is that it

This work is supported in part by NSF IIS-096053 and Autodesk.

Both authors are with Department of Computer Science, George Mason University, 4400 University Drive MSN 4A5, Fairfax, VA 22030 USA, \{ebehar, jmlien\}@cs. gmu. edu

\author{
Jyh-Ming Lien
}

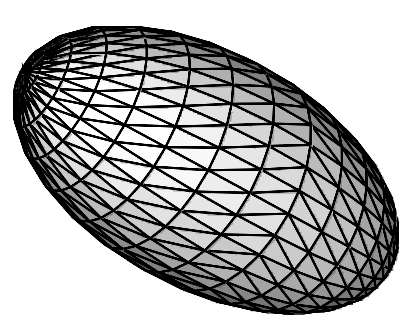

(a) ellipse

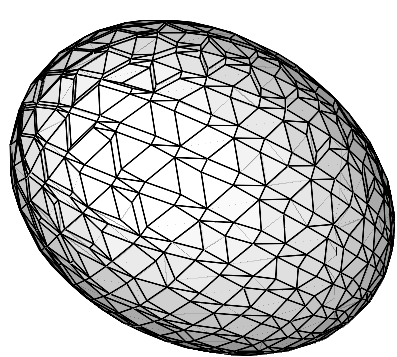

(c) (a) $\oplus \mathrm{GS} 4$

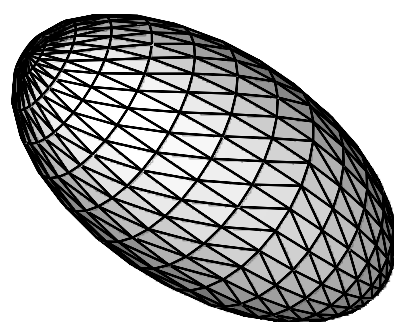

(b) slightly rotated ellipse

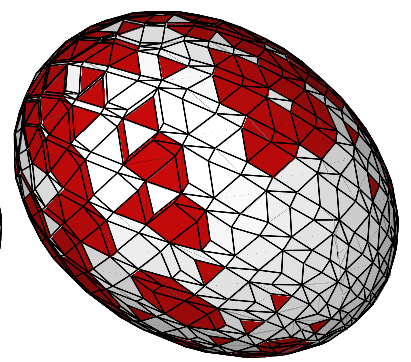

(d) (a) $\oplus$ GS4
Fig. 1. The Minkowski sums of a rotating ellipse and a sphere (GS4 shown in Fig. 3). The ellipse in (b) is rotated by $\pi / 40$ from (a). The dark (red) facets in (d) are the differences between (c) and (d).

requires the same amount of computation even when a small amount of rotation is applied to $P$ or $Q$.

Our work is motivated by the observation described above. Thus, our objective is to compute the Minkowski sums of rotating convex polyhedra without re-computing the entire Minkowski sum repetitively. The main idea in our method is to generate the Minkowski sum from the existing Minkowski sum. More specifically, we generate the new Minkowski sum by correcting the "errors" introduced by rotation.

In theory, computing the Minkowski sum of two convex shapes $P$ and $Q$ will take $O(m n \log m n)$ time by overlaying the Gaussian maps of $P$ and $Q$ with complexities $O(m)$ and $O(n)$, respectively [3]. It is also known that the (space) complexity of the Minkowski sum of the same $P$ and $Q$ is $O(m n)$ [3]. Therefore, we expect an algorithm, similar to ours, that updates the Minkowski sum, instead of recomputing from scratch, will be $O(\log m n)$ faster than the traditional (brute force) approach.

Our Contribution. In this paper, we propose a method that provides the desired properties mentioned above. We call this method: DYMSUM (dynamic Minkowski sum). We show that DYMSUM is significantly more efficient than the naïve method of re-computing the Minkowski sum from scratch, in particular when the size of the input polyhedra are large and when the rotation angle is small between frames. From 
our experimental results, we show that the computation time of DYMSUM grows slowly (e.g., linearly if inputs are cubes) with respect to the size of the input comparing to the naïve approach (see Section V). A preliminary version of this work can be found in a video abstract [9]. Although we focus on convex shapes, DYMSUM can be used as the basic operation for non-convex polyhedra using convex decomposition.

\section{RELATED WORK}

Many methods have been proposed to compute the Minkowski sums of polygons or polyhedra, however, to the best of our knowledge, Mayer et al. [10] and our preliminary work [9] are the only works focusing on the Minkowski problem involving rotating polyhedra.

Mayer et al. [10] observed that the combinatorial structure of the Minkowski sum given two such polyhedra only changes at certain critical rotation values, and construct an efficient search structure they call a criticality map. Unfortunately, as they address in the paper, even for convex polyhedra with moderate numbers of vertices, the criticality map can become very large and costly to construct. They present concepts of the criticality map for two and three axes of rotation, but the growth order on the size of these structures makes them expensive to build and prohibitive to store. Enhancements are presented for using an axis-angle representation to answer general rotation queries, as well as dynamic processing to reduce the size of the structure, which trades off query time to reduce build time and storage requirements for the criticality map.

In order to provide enough background in hope that the readers can appreciate the results of this work more, we briefly review existing works on the Minkowski sum of static inputs; see more detailed surveys in [1], [2], [3].

Convolution. The convolution of two shapes $P$ and $Q$, denoted as $P \otimes Q$, is a set of line segments in 2-d or facets in 3-d that is generated by 'combining' the segments or the facets of $P$ and $Q$ [11]. One can think of the convolution as the Minkowski sum that involves only the boundary, i.e., $P \otimes Q=\partial P \oplus \partial Q$. It is known that the convolution forms a superset of the Minkowski sum [1], i.e., $\partial(P \oplus Q) \subseteq P \otimes Q$. To obtain the Minkowski sum boundary, it is necessary to trim the line segments or the facets of the convolution.

For 2-d polygons, Guibas and Seidel [12] show an output sensitive method to compute convolution curves. Later, Ghosh [1] proposed an approach, which unifies 2-d and $3-d$, convex and non-convex, and Minkowski addition and decomposition operations. The main idea in his method is the negative shape and slope diagram. The slope diagram is closely related to the Gaussian map, which has been recently used by Fogel and Halperin [3] to implement robust and efficient Minkowski sum computations of convex objects.

The main difficulty of the convolution-based methods is in removing the portions of the facets that are inside the Minkowski sum. Recently, Wein [13] showed a robust and exact method based on convolution for non-convex polygons. To obtain the Minkowski sum boundary from the convolution, his method computes the arrangement induced by the line segments of the convolution and keeps the cells with non-zero winding numbers. No practical implementation is known for polyhedra using convolution due to the difficulty of computing the 3-d arrangement and its substructures [14].

Convex decomposition. In the convex decomposition framework, the input models are decomposed into convex components. Then, this framework computes the pairwise Minkowski sums of the components. Finally, all these pairwise Minkowski sums are merged.

The decomposition approach was first proposed by Lozano-Pérez [15] to compute $\mathcal{C}$-obst for motion planning. Although the main idea of this approach is simple, the decomposition step and the merge step (i.e., union) can be very tricky. For example, it is known that creating solid convex decomposition robustly is difficult [16]. In addition, Agarwal et al. [17] have shown that different decomposition strategies can greatly affect the efficiency of this approach. Hachenberger [18] presents a robust and exact implementation using the Nef polyhedra in CGAL. However, his results are still only practical for simple models.

The union step is even more troublesome. Flato [19] computes the union using the cells induced by the arrangement of the line segments. He uses a hybrid strategy that combines arrangement with incremental insertion to gain better efficiency. Hachenberger [18] also studies how the order of the union operation affects the efficiency. To avoid this explicit union step, Varadhan and Manocha [2] proposed an approach that generates meshes approximating the Minkowski sum boundary. Marching cube techniques are used to extract the iso-surface from a signed distance field. They also proposed an adaptive cell to improve the robustness and efficiency of their method.

Point-Based Representation. Alternatively, points have been used to represent the Minkowski sum boundary [20]. Representing the boundary using only points has many benefits. First of all, generating such points is easier than generating meshes and can be done in parallel and in multi-resolution fashion [21]. Moreover, point-based representations can be generalized to higher dimensional motion planning problems [22].

Voxelization. Li and McMains [23] propose a GPU-based voxelization technique which directly computes a volumetric solid with high accuracy instead of an exact boundary representation of the Minkowski sum. The main limitation is that this technique does not generate the inner holes of the Minkowski sum.

\section{A BRUte Force Method}

Without loss of generality, we assume that $P$ is movable while $Q$ is stationary. We let $P_{s}$ and $P_{t}$ be two copies of $P$ at two configurations $s$ and $t$ with distinct orientations. Our goal is to compute $M_{t}=P_{t} \oplus Q$ from $M_{s}=P_{s} \oplus$ $Q$. Moreover, the computation time of the Minkowski sum should be sensitive to the orientation difference between $P_{s}$ and $P_{t}$, i.e., the smaller the difference between $P_{s}$ and $P_{t}$, the faster the computation of $M_{t}$. 
Computing the Minkowski sum of two convex shapes is usually based on the idea of overlaying two Gaussian maps of the inputs. The Gaussian map $g(P)$ of a polyhedron $P$ is a sub-division of $\mathbb{S}^{2}$. One can think $g(P)$ and $P$ as dual to one another. That is, each face $f$ of $P$ with the outward normal $n_{f}$ corresponds to a vertex $g(f) \in g(P)$ with coordinate $n_{f}$, and each vertex $v$ of $P$ corresponds to a face $g(v) \in g(P)$ bounded by the normals of the faces incident to $v$. When we overlay two Gaussian maps $g(P)$ and $g(Q)$, a vertex $v$ in $g(P)$ must be associated with exactly one face in $g(Q)$ that encloses $v$ and vice versa. Moreover, the edges in $g(P)$ and $g(Q)$ can also intersect.

The facets of a Minkowski sum are defined exactly by these two types of interactions between $g(P)$ and $g(Q)$ : the facets generated from a facet of $P$ and a vertex of $Q$ or vice versa, called $f v$-facets; and the facets generated from a pair of edges from $P$ and $Q$, respectively, called $e e$-facets. A facet $f$ and a vertex $v$ produce an $f v$-facet if and only if the normal of $f$ is a convex combination of the normals of the facets incident to $v$. Similarly, a pair of edges $e_{1}$ and $e_{2}$ form an $e e$-facet if and only if the cross product of vectors parallel to $e_{1}$ and $e_{2}$ is a convex combination of the normals of the facets incident to $e_{1}$ and $e_{2}$.

These criteria allow us to test if a given pair of features (a facet/vertex pair or an edge pair) will produce a Minkowski sum facet by checking only the neighborhood of these features. Given a pair of features (facet/vertex or edge/edge), we say that the features are compatible if they form either an $f v$-facet or an $e e$-facet. When $P_{s}$ transforms to $P_{t}$, some facets (i.e., pairs of features) in $M_{s}$ will no longer be compatible. We call these facets the "errors" introduced by rotation.

A brute force algorithm, which is used in all existing methods except [10], computes the Minkowski sums from $P_{s}$ and $P_{t}$ without considering the correspondences between them as shown in Algorithm III.1. Given $P_{s}$ and $Q$ and the existing Minkowski sum $M_{s}$. Algorithm III.1 rotates $P_{s}$ by $\theta$ to obtain $P_{t}$. Then it uses an existing Minkowski sum algorithm to compute $M_{t}$.

\section{Algorithm III.1: $\operatorname{BruteForce}\left(M_{s}, P_{s}, Q, \theta\right)$}

$$
\begin{aligned}
& P_{t}=\operatorname{Rotate}\left(P_{s}, \theta\right) \\
& M_{t}=\operatorname{MinkowskiSum}\left(P_{t}, Q\right) \\
& \text { return }\left(M_{t}\right)
\end{aligned}
$$

\section{DYNAMIC MINKOWSKI SUMS (DYMSUM)}

In this section, we describe the details of the proposed method DYMSUM. Our goal is to take advantage of the correspondences between $M_{s}$ and $M_{t}$ that are completely ignored by Algorithm III.1. Let us consider the Gaussian map again. $M_{s}$ is computed by overlaying $g\left(P_{s}\right)$ and $g(Q)$. To obtain $M_{t}$, we need to find out which vertices in $g\left(P_{s}\right)$ are moved to another face in $g(Q)$ and determine whether the edges of $g\left(P_{s}\right)$ intersect or stop intersecting with the edges of $g(Q)$ after rotating $P_{s}$ to $P_{t}$. This is exactly what DYMSUM does. That is, DYMSUM first determines these changes in the overlay introduced by the rotation, and then corrects the errors to generate the new Minkowski sum $M_{t}$. Therefore, the Minkowski sum $M_{t}$ is composed of two types of facets: (1) the facets from $M_{s}$ that still satisfy the aforementioned criteria after rotation and (2) the facets that are created due to the errors.

A sketch of DYMSUM is shown in Algorithm IV.1. In contrast to the brute-force method, DYMSUM is sensitive to the amount of rotation. That is, when $\theta$ is smaller, there will be fewer errors in the Gaussian map overlay. In this case, DYMSUM will likely take less time to compute the result than the naïve method. In the rest of this section, we will discuss how the errors are determined (Section IV-A) and how to correct these errors (Sections IV-B and IV-C).

Algorithm IV.1: $\operatorname{DYMsum}\left(M_{s}, P_{s}, Q, \theta\right)$

$$
\begin{aligned}
& P_{t}=\operatorname{Rotate}\left(P_{s}, \theta\right) \\
& E_{t}=\text { FindErrors }\left(M_{s}, P_{t}, Q\right) \\
& M_{t}=\operatorname{CorrectErrors}\left(E_{t}, M_{s}, P_{t}, Q\right)
\end{aligned}
$$$$
\text { return }\left(M_{t}\right)
$$

\section{A. Find Errors}

There are two types of errors, $f v$-errors and $e e$-errors, corresponding to $f v$-facets and $e e$-facets, respectively. If a pair of features was compatible and becomes incompatible after the rotation of $P$, we call this pair an error.

Before we talk about how these errors can be identified, we will first show the relationship between the $f v$-errors and the ee-errors. Theoretically, the complexity of the Minkowski sum is $O(m n)$ and there can only be $O(m+n) f v$-facets. Therefore, the number of $f v$-facets can be far smaller than the number of $e e$-facets. Moreover, it is easy to show that no $e e$-errors can occur if there are no $f v$ errors.

Theorem 4.1: $f v$-errors and $e e$-errors must coexist.

Proof: We first show that if there is an ee-error, there must be an $f v$-error. Let $e$ and $e^{\prime}$ be a pair of edges that are compatible before rotation and become an ee-error after rotation. When $e$ and $e^{\prime}$ are compatible, $g(e)$ and $g\left(e^{\prime}\right)$ must intersect and, after rotating $P, g(e)$ and $g\left(e^{\prime}\right)$ no longer intersect. This means at a certain point during the rotation, an end point of $g(e)$ must cross $g\left(e^{\prime}\right)$ or vice versa. When a point $v$ crosses the edge $g(e), v$ changes the face with which it is associated from one side of $g(e)$ to the other side of $g(e)$. This change indicates that there must be an $f v$-error.

We then show that if there is an $f v$-error, there must be an ee-error. If a facet $f$ of $P$ and a vertex $v$ of $Q$ become an $f v$-error, we know that $f$ now must be compatible with some other vertex $v^{\prime} \neq v$ of $Q$. As a result, an edge $g(e)$ incident to $g(f)$ must be moved (or deformed) with $g(f)$. Since the faces in $g(Q)$ are convex and $g(e)$ cannot intersect with a segment more than twice, $g(e)$ must intersect with some new edges of $Q$ when $g(f)$ moves from $g(v)$ to $g\left(v^{\prime}\right)$. This indicates that there must be at least one $e e$-error.

Therefore, $f v$-errors and $e e$-errors must coexist. 
Based on Theorem 4.1, we can find all errors by first exhaustively checking all $f v$-facets in $M_{s}$ (the Minkowski sum before rotation) to find $f v$-errors. Then we use these $f v$-errors to identify all $e e$-errors. That is, if there are no $f v$ errors found, then we can immediately conclude that there are no $e e$-errors as well. Otherwise, the ee-errors must occur at the edges incident to the vertices involved in the $f v$-errors. Thus, it is clear that finding all $f v$-errors will take $O(m+n)$ time.

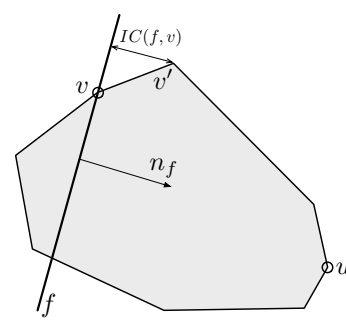

(a) degree of incompatibility

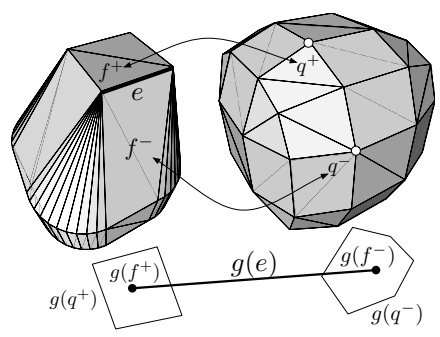

(b) correct an ee-error
Fig. 2. (a) A 2-d cartoon shows the definition of $I C(f, v)$ and its witness $\overline{v v^{\prime}}$. Using gradient decent, we will find the compatible vertex $u$ for $f$. (b) Determine the associated edges for edge $e$.

\section{B. Correct fv-errors}

For each $f v$-error, we perform a gradient descent to compute a new $f v$-facet. More specifically, given a facet/vertex pair, we can measure the degree of incompatibility of the pair and attempt to iteratively minimize the incompatibility until a compatible pair is found.

Let $f$ be a facet of $P$ and $v$ be a vertex of $Q$. When $f$ and $v$ are compatible, all the edges that are incident to $v$ must be below or on the half-plane supported by $f$. When $f$ and $v$ are incompatible, we can define the degree of incompatibility

$$
I C(f, v)=\max \left\{\mathrm{d}(e, f) \mid e \in E_{v}\right\},
$$

where $E_{v}$ is a set of edges incident to $v$ and $\mathrm{d}(e, f)$ is the longest Euclidean distance from any point on $e$ to $f$. We say an edge $e$ is the witness of the incompatibility if $\mathrm{d}(e, f)$ is $I C(f, v)$. Fig. 2(a) illustrates an example of $f v$-error and $I C(f, v)$.

In order to find the compatible pair, we find the witness of the incompatibility $e$, and replace $v$ with the other end point $v^{\prime} \neq v$ of $e$, and repeat this until $f$ and $v$ become compatible. In Fig. 2(a), this vertex is $u$. Since $Q$ is convex, this procedure must be able to find a vertex of $Q$ such that all of its incident edges are below $f$, therefore, will always terminate.

This process is equivalent to finding an extreme point at the outward normal direction of $f$ and therefore can be done in $O(\log n)$ time if $Q$ has $n$ vertices.

\section{Correct ee-errors}

After all the $f v$-errors are corrected, the incident edges associated with these $f v$-errors are marked as ee-errors. Let $e$ be such an edge from $P$ and let $f^{-}$and $f^{+}$be the facets in $P$ incident to $e$. Our goal is to find the edges of $Q$ that are compatible with $e$. An exhaustive search for compatible edges will certainly be slow. Fortunately, we can find the compatible edges using the results from $f v$-facets. That is, since we know that the incident facets $f^{-}$and $f^{+}$both have the compatible vertices $q^{-}$and $q^{+}$of $Q$, we can find the compatible edges for $e$ using $q^{-}$and $q^{+}$. The relationships between $e, f^{ \pm}$and $q^{ \pm}$are shown in Fig. 2(b).

More specifically, if we overlay the Gaussian map $g(e)$ of $e$ with $g(Q), g(e)$ will intersect a set of faces in $g(Q)$ and the end points of $g(e)$ are inside $g\left(q^{-}\right)$and $g\left(q^{+}\right)$. See the bottom of Fig. 2(b). If we can determine the rest of the faces intersected by $g(e)$, we can find the compatible edges for $e$. We further know that these faces form a connected component between $g\left(q^{-}\right)$and $g\left(q^{+}\right)$, thus the compatible edges for $e$ must be on the boundary of these faces. To find these Gaussian faces, we start from $g\left(q^{-}\right)$, and find an incident edge $e^{\prime}$ of $g\left(q^{-}\right)$that is compatible with $e$. It is obvious that $e^{\prime}$ must exist unless $q^{-}=q^{+}$. From $e^{\prime}$, we replace $q^{-}$with the vertex $x^{\prime} \neq q^{-}$incident to $e^{\prime}$, and repeat the process until $q^{-}=q^{+}$.

The computation time is equal to the sum of the degree of vertices of $Q$ visited during the search process.

\section{EXPERIMENTAL RESULTS}

In this section, we show that the computation time of DYMSUM is more efficient than the traditional approach (Algorithm III.1) and is indeed sensitive to the amount of rotation applied to $P$. In our experiments, the polyhedron $P$ rotates using a sequence of random quaternions. Each quaternion is applied to $P$ for a random period of time. All the computation times that we show below are obtained by averaging over 100 random rotations. All the experiments are performed on a machine with Intel CPUs at $2.13 \mathrm{GHz}$ with 4 GB RAM. Our implementations are coded in $\mathrm{C}++$.

Figure 3 shows 13 models that we use in the first two experiments. Many of these models are from [3] and can be obtained from the authors' website. Theoretically, DYMSUM works with polyhedra tessellated with arbitrary polygons, but in our current implementation DYMSUM only takes triangulated polyhedra. Therefore, all the models in Figure 3 are triangulated.

\section{A. Experiment 1: Dymsum vs. Brute-force method}

In Table I, we compare the proposed method, DYMSUM, to a brute-force method (Algorithm III.1) that re-computes the Minkowski sum in every time step. The brute-force method checks the compatibility of all facet-vertex and edge-edge pairs every time that $P$ rotates. The values in the table are $t_{d} / t_{b f}$, where $t_{d}$ and $t_{b f}$ are the (averaged) updating or recomputing times for DYMSUM and the brute-force method.

From Table I, it is clear that DYMSUM is always faster than the brute-force method. Even for very simple cases, such as cone $\oplus$ HP, DYMSUM is at least 8 times faster. For more complex examples, such as ellipse $\oplus$ ellipse, DYMSUM is about 176 times faster than the brute-force method. 
TABLE I

THE SPEEDUP OF DYMSUM USING THE MODELS IN FIG. 3.

The values in the table are $t_{d} / t_{b f}$, where $t_{d}$ and $t_{b f}$ are the computation times for DYMSUMand the brute-force method.

\begin{tabular}{|c|c|c|c|c|c|c|c|c|c|c|c|c|c|}
\hline cone & 9.26 & & & & & & & & & & & & \\
\hline cube & 8.58 & 10.50 & & & & & & & & & & & \\
\hline cylinder & 12.83 & 10.31 & 18.73 & & & & & & & & & & \\
\hline DD & 9.82 & 9.85 & 13.33 & 10.93 & & & & & & & & & \\
\hline ellipse & 24.97 & 14.50 & 47.51 & 21.67 & 176.34 & & & & & & & & \\
\hline GS1 & 15.09 & 11.21 & 23.66 & 15.39 & 51.38 & 25.23 & & & & & & & \\
\hline GS2 & 19.73 & 12.15 & 32.96 & 17.95 & 86.99 & 33.16 & 52.73 & & & & & & \\
\hline GS3 & 22.92 & 12.42 & 40.45 & 19.63 & 120.23 & 38.88 & 65.53 & 86.26 & & & & & \\
\hline GS4 & 24.50 & 12.70 & 45.22 & 20.19 & 146.15 & 44.21 & 73.89 & 100.15 & 121.26 & & & & \\
\hline HP & 8.02 & 9.32 & 9.59 & 9.25 & 13.19 & 9.75 & 10.84 & 11.58 & 11.51 & 9.00 & & & \\
\hline $\mathrm{T}$ & 14.50 & 11.16 & 20.66 & 14.05 & 35.95 & 20.33 & 26.01 & 29.21 & 31.83 & 10.28 & 16.17 & & \\
\hline TI & 21.30 & 13.87 & 35.55 & 19.53 & 91.48 & 38.02 & 56.86 & 69.25 & 78.26 & 12.78 & 28.08 & 63.45 & \\
\hline v-rod & 20.30 & 18.31 & 34.00 & 21.92 & 88.30 & 46.53 & 65.46 & 77.73 & 83.05 & 15.76 & 34.23 & 67.24 & 123.75 \\
\hline & cone & cube & cylinder & $\overline{\mathrm{DD}}$ & ellipse & $\overline{\text { GS1 }}$ & $\overline{\mathrm{GS} 2}$ & $\overline{\mathrm{GS} 3}$ & $\overline{\mathrm{GS} 4}$ & HP & $\bar{T}$ & TI & v-rod \\
\hline
\end{tabular}

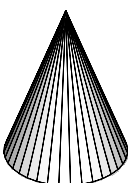

(a)

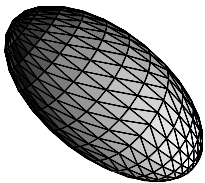

(e)

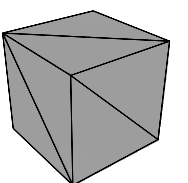

(b)

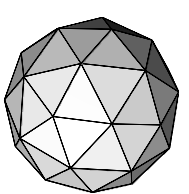

(f)

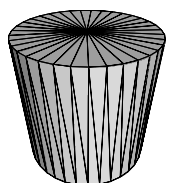

(c)

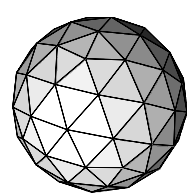

(g)

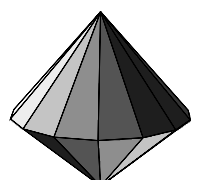

(d)

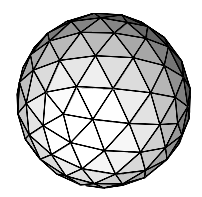

(h)

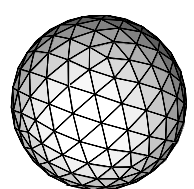

(i)

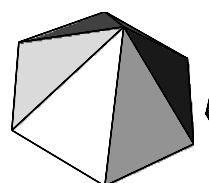

(j)

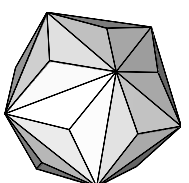

(k)

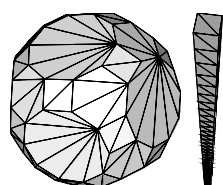

(1) around $x=500$ and then stabilizes below the 0.5 millisecond mark. In Experiments 1 and 3, we set $x=500$.

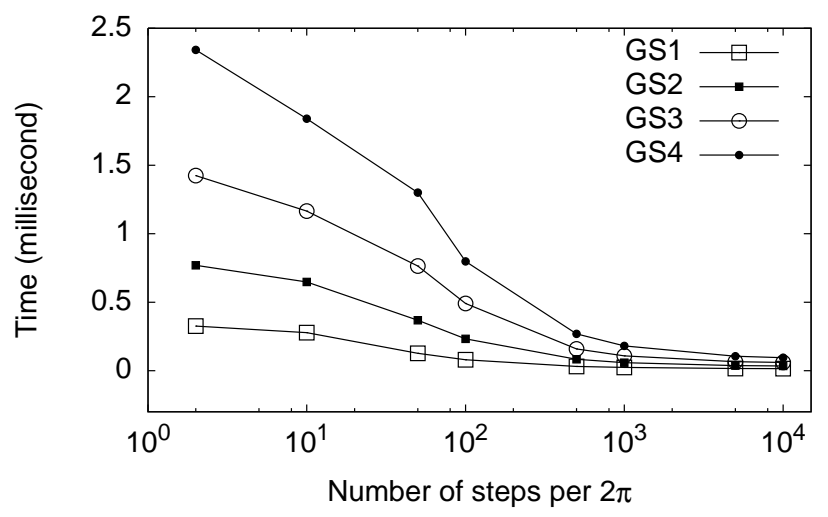

Fig. 4. Computation time at different rotational speeds. More steps per $2 \pi$ means slower rotational speed.

Fig. 3. Models used in the experiments. (a) cone, 78 facets (b) cube, 12 facets (c) cylinder, 140 facets (d) dioctagonal dipyramid (DD), 32 facets (e) ellipse, 960 facets (f) geodesic sphere 1 (GS1), 80 facets (g) GS2, 180 facets (h) GS3, 320 facets (i) GS4, 500 facets (j) hexagonal pyramid (HP), 10 facets $(\mathrm{k})$ triakis icosahedron $(\mathrm{T}), 60$ facets $(\mathrm{l})$ truncated icosidodecahedron (TI), 236 facets (m) v-rod, 324 facets.

\section{B. Experiment 2: Computation time vs. Rotational resolution}

In this experiment, we study the computation time of DYMSUM with respect to the rotational resolution of $P$. Our goal is to show that, in contrast to the brute force approach, DYMSUM is in fact sensitive to the magnitude of the rotation. In the problem of motion planning, this resolution defines the number of slices in mapping the configuration space. In the physically-based simulation, this value defines the number of collision detections and penetration depth estimations per second. Fig. 4 shows the results obtained using DYMSUM. Notice that the $x$ axis is in logarithmic scale.

The $x$ axis of Fig. 4 is the number of steps for $P$ to make a full rotation. For example, when $x=500, P$ will take 500 steps to rotate $360^{\circ}$ degree. That is, $P$ rotates $\pi / 250$ around a random axis every step. Therefore, when $x$ is large, the changes in the Minkowski sum will be small. From the figure, we can see that the computation time drops quickly

\section{Experiment 3: Computation time vs. Input size}

In this last experiment, we study the relationship between the computational time and the input size. We use a $10 \times 10 \times$ 10 cube with different numbers of triangles tessellated on the surface. Fig. 5 shows that the computation time of the bruteforce method increases rapidly while that of DYMSUM stays almost constant. When we show DYMSUM's computation time along in Fig. 5(b), DYMSUM's computation time is increased linearly along with the size of the cubes.

Recall that the complexity of a Minkowksi sum of two convex shapes is $O(m n)$, however the number of the $f v$ facets is $O(m+n)$. Therefore a large portion of the Minkowksi sum is composed of the ee-facets. In our experiment, we see a linear increase in computation time. We speculate that only a few errors occur at each step and most of the computation time is spent on verifying and updating the compatibility of the $f v$-facets.

\section{CONCLUSION AND DISCUSSION}

We have shown an efficient and robust method for recomputing 3-d Minkowski sums of convex polyhedra under rotation. Experimentally, we have shown that DYMSUM 


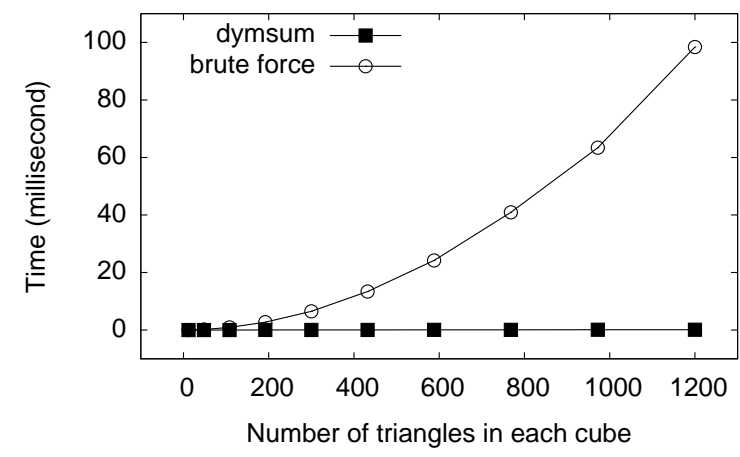

(a) DYMSUM vs. brute force

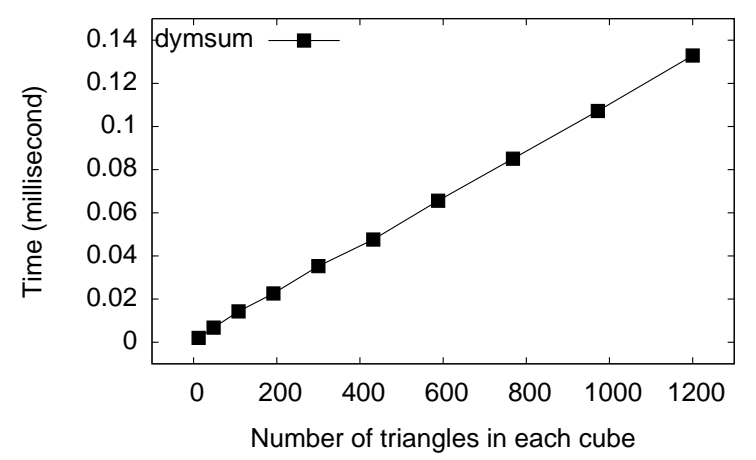

(b) DYMSUM only

Fig. 5. Computation times of DYMSUM and brute force of two identical cubes. The numbers of triangles in the cubes are 12, 48, 108, 192, 300, 432, 588, 768, 972 and 1200.

greatly outperforms the brute-force method of re-computing the Minkowski sum from scratch at each iteration.

Also, because the performance of the algorithm increases as the magnitude of the rotation between steps decreases, this method is much more useful than the naïve approach for generating $\mathcal{C}$-obst using discretized rotation intervals, as it enables the fast computation of higher resolution $\mathcal{C}$-obst approximations.

The main steps in our method are those of identifying and rectifying errors introduced by rotation. It is possible to efficiently compute this because of the observation that there are few $f v$-errors, and that $e e$-errors propagate locally to corresponding $f v$-errors. Instead of re-computing the entire Minkowski sum at a cost of $O(m n \log n)$ time, we are able to rectify the errors in significantly less time, which is the major efficiency gain from our method.

We hope to extend this method in the future to work with non-convex polyhedra. We also plan to apply this method to general $\mathcal{C}$-space mapping for convex polyhedra.

\section{REFERENCES}

[1] P. K. Ghosh, "A unified computational framework for Minkowski operations," Computers and Graphics, vol. 17, no. 4, pp. 357-378, 1993.

[2] G. Varadhan and D. Manocha, "Accurate Minkowski sum approximation of polyhedral models," Graph. Models, vol. 68, no. 4, pp. 343-355, 2006.

[3] E. Fogel and D. Halperin, "Exact and efficient construction of Minkowski sums of convex polyhedra with applications," in Proc. 8th Wrkshp. Alg. Eng. Exper. (Alenex'06), 2006, pp. 3-15.

[4] A. Kaul and J. Rossignac, "Solid-interpolating deformations: construction and animation of PIPs," in Proc. Eurographics '91, 1991, pp. 493-505.

[5] P. Gritzmann and B. Sturmfels, "Minkowski addition of polytopes: computational complexity and applications to Gröbner bases," SIAM J. Discret. Math., vol. 6, no. 2, pp. 246-269, 1993.

[6] K. Fukuda, "From the zonotope construction to the minkowski addition of convex polytopes," Journal of Symbolic Computation, vol. 38, no. 4, pp. 1261-1272, 2004

[7] L. Zhang, Y. J. Kim, G. Varadhan, and D. Manocha, "Generalized penetration depth computation," Comput. Aided Des., vol. 39, no. 8, pp. 625-638, 2007.
[8] K. Wise and A. Bowyer, "A survey of global configuration-space mapping techniques for a single robot in a static environment," The International Journal of Robotics Research, vol. 19, no. 8, pp. 762779, 2000.

[9] J.-M. Lien, "Minkowski sums of rotating convex polyhedra," in Proc. 24th Annual ACM Symp. Computat. Geom. (SoCG), June 2008, video Abstract.

[10] N. Mayer, E. Fogel, and D. Halperin, "Fast and robust retrieval of minkowski sums of rotating polytopes in 3-space," in To appear in Proc. Symposium of Solid and Physical Modeling (SPM), 2010.

[11] L. J. Guibas, L. Ramshaw, and J. Stolfi, "A kinetic framework for computational geometry," in Proc. 24th Annu. IEEE Sympos. Found. Comput. Sci., 1983, pp. 100-111.

[12] L. J. Guibas and R. Seidel, "Computing convolutions by reciprocal search," Discrete Comput. Geom., vol. 2, pp. 175-193, 1987.

[13] R. Wein, "Exact and efficient construction of planar Minkowski sums using the convolution method," in Proc. 14th Annual European Symposium on Algorithms, 2006, pp. 829-840.

[14] S. Raab, "Controlled perturbation for arrangements of polyhedral surfaces with application to swept volumes," in SCG '99: Proceedings of the fifteenth annual symposium on Computational geometry. New York, NY, USA: ACM, 1999, pp. 163-172.

[15] T. Lozano-Pérez, "Spatial planning: A configuration space approach," IEEE Trans. Comput., vol. C-32, pp. 108-120, 1983.

[16] C. Bajaj and T. K. Dey, "Convex decomposition of polyhedra and robustness,” SIAM J. Comput., vol. 21, pp. 339-364, 1992.

[17] P. K. Agarwal, E. Flato, and D. Halperin, "Polygon decomposition for efficient construction of minkowski sums," in European Symposium on Algorithms, 2000, pp. 20-31.

[18] P. Hachenberger, "Exact Minkowksi sums of polyhedra and exact and efficient decomposition of polyedra in convex pieces," in Proc. 15th Annual European Symposium on Algorithms (ESA), 2007, pp. 669680.

[19] E. Flato, "Robuts and efficient construction of planar minkowski sums," M.Sc. Thesis, Dept. Comput. Sci., Tel-Aviv Univ., Isael, 2000.

[20] M. Peternell, H. Pottmann, and T. Steiner, "Minkowski sum boundary surfaces of 3d-objects," Vienna Univ. of Technology, Tech. Rep., August 2005.

[21] J.-M. Lien, "Point-based minkowski sum boundary," in $P G$ '07: Proceedings of the 15th Pacific Conference on Computer Graphics and Applications. Washington, DC, USA: IEEE Computer Society, 2007, pp. 261-270.

[22] — "Hybrid motion planning using Minkowski sums," in Proc. Robotics: Sci. Sys. (RSS), Zurich, Switzerland, 2008.

[23] W. Li and S. McMains, "A gpu-based voxelization approach to $3 \mathrm{~d}$ minkowski sum computation," in SPM '10: Proceedings of the 14th ACM Symposium on Solid and Physical Modeling. New York, NY, USA: ACM, 2010, pp. 31-40. 